\title{
Resident Duty Hours: A Review
}

Lindsay Melvin MD, Sophie Corriveau MD, Aiman Alak MD, Ameen Patel MD

\begin{abstract}
About the Authors
Lindsay Melvin is a PGY4 resident in General Internal Medicine at the University of Toronto. Sophie Corriveau is a PGY4 resident in Respirology at McMaster University. Aiman Alak is a PGY4 resident in Cardiology at McMaster University. Ameen Patel is a staff member of the Division of General Internal Medicine and holds the William J. Walsh Chair in Medical Education, all at McMaster University, in Hamilton, Ontario. Correspondence may be directed to sophie.corriveau@medport.ca
\end{abstract}
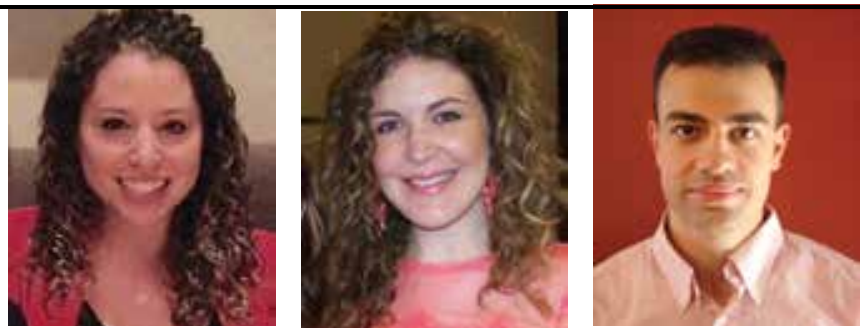

$\mathrm{R}$ esidents are physicians undertaking further training to become independently licensed practitioners. Historically, resident duty hour periods were long and intense. The goal was to maximize learning through high patient volume and to teach doctors how to take responsibility. Recently, concerns over patient and resident safety have led to restricted trainee work hours. The putative justification is to improve resident education, resident well-being, and patient care. In light of this recent shift in the medical culture, resident duty hours have become a controversial topic.

Restricted duty hours take many forms. In the United States, the Accreditation Council for Graduate Medical Education (ACGME) mandated junior residents work no longer than 16 consecutive hours, while senior residents could work up to 26 hours. ${ }^{1}$ In Canada, no nationwide mandate exists and the issue falls within provincial jurisdiction. In Ontario, under the Professional Association of Residents of Ontario agreement, call-periods are no more than 26 consecutive hours in-house, no more than one in four nights in-house, or no more than one in three nights of home-call. After a 2011 Quebec court ruling, resident duty hours were restricted to 16 consecutive hours in that province. This resulted from the court concluding that traditional hours violate the Canadian Charter of Rights and Freedoms. Regardless, the Quebec ruling prompted other Canadian programs to further reduce resident duty hours and consecutive hours on-call.

To better understand this complex issue, the following review discusses resident safety, resident performance, resident education, and patient safety. Our goal is to present a balanced, evidence-based discussion, addressing both patient safety and resident fatigue management.

\section{Resident Safety}

In 2005, Barger and colleagues conducted a nationwide webbased survey in which 2737 residents completed monthly reports about work hours, motor vehicle accidents, and near misses. In a prospective analysis, every extended work shift that was scheduled in a month increased the monthly risk of a motor vehicle crash by $9.1 \%$ (95\% CI 3.4-14.7\%). ${ }^{2}$ The results, which were mirrored in another study, raised concerns about the effects of sleep deprivation on resident safety. ${ }^{3}$

\section{Sleep and Resident Performance}

In addition to personal safety, the performance of sleep-deprived residents may be worse. Arnedt and colleagues evaluated training-related performance impairment against an accepted standard of functional impairment. ${ }^{4}$ In this prospective study, 34 pediatrics residents were tested following heavy call rotations and while presumably sleep-deprived. Residents on lighter rotations were tested in the same domains after ingesting alcohol. The authors found significant decrements in attention, vigilance, and simulated-driving performance in the sleep deprived cohort. These impairments were similar to well-rested residents with a 0.04- $0.05 \%$ blood-alcohol level. In Canada, a blood alcohol level of 0.05 can result in roadside license suspension, while over 0.08 is a criminal offense.

Lingenfelser and colleagues found electrocardiogram reading, short-term memory recall, and reaction times all deteriorated after 24 hours on call. ${ }^{5}$ These deficits were similar among junior and senior residents, suggesting a lack of adaption through residency training. While studies have suggested that sleep deprivation negatively impacts cognitive tasks and performance, this has not been linked with poor patient care. 


\section{Effect of Duty-Hour Restriction on Patient Outcomes}

Overall, there is no clear signal that reducing resident duty hours impacts patient safety. For example, a systematic review in 2004 examined patient mortality, adverse events, and medication errors. ${ }^{6}$ This systematic review, though limited by the quality of the studies available, found no change in patient mortality after the implementation of reduced working hours for residents.

A systematic review published in the Annals of Internal Medicine examined studies involving residency shifts with defined length, night float, or protected sleep. Only one of three studies found that six-month patient mortality reduction was associated with limited duty hours. However, all studies demonstrated reduced medical errors where resident duty hours were limited. ${ }^{7}$

The most recent systematic review of 135 studies by Ahmed and colleagues examined both night-float and 16-hour shift systems separately. ${ }^{8}$ There was no overall improvement in patient outcomes attributable to resident duty hour reduction; however, several studies found increased complication rates with reduced resident duty hours for high acuity. ${ }^{8}$

\section{Effect of Duty Hour Restriction on Education}

Exposure and intensity is crucial for learning. However, the growing ease with which information can be accessed, and the growth of simulation may mitigate this concern. Goitein and colleagues studied the overall effect of duty hour restriction on resident education and found that more residents reported a negative effect (47\%) than positive $(32 \%)$ or neutral $(21 \%) .{ }^{9}$ Seventy percent of residents felt there was not enough time for teaching by attending physicians and formal education was frequently missed. ${ }^{9}$ Overall, senior residents were less likely to approve of duty hour restrictions and more likely to report negative effect on education. These findings were confirmed by a systematic review by Ahmed and colleagues. ${ }^{8}$

\section{Effect of Duty-Hour Restriction on Resident Well-Being}

Overall, studies into the associations between resident duty hours and resident well-being are inconclusive. In the general population, the 12-month prevalence of any mood disorder is $9.5 \%$. By comparison, studies consistently demonstrate a depression prevalence of $27-30 \%$ within 12 months of residency training. ${ }^{10}$ Reduced duty hours can lead to work compression, namely, trying to accomplish the same tasks in a reduced period of time. This, in turn, can increase rather than alleviate pressure and stress for residents. ${ }^{11}$ While the implementation of the 80 hour work-week with the first ACGME mandate in 2003 was followed by improvement in resident wellness, reducing the shift length to 16-hour maximums in 2011 was not associated improved resident experiences. ${ }^{8}$

The well-publicized study by Sen and colleagues further illustrates the conflicting nature of duty hour reform. ${ }^{12}$ The group studied the effects of the 2011 duty-hour reforms in over 2000 United States residents regarding sleep, work hours, and medical errors, There was no change in hours slept, depression scores, or subjective well-being scores following a limited change in resident duty hour conditions (16-hour maximums). However, the average weekly duty hours only decreased from 67 hours to 64 hours. ${ }^{12}$

\section{Duty-Hour Restriction and Handover}

One possible reason that patient safety has not improved by reducing resident duty hours is because this creates more handovers and between relatively junior practitioners. Many studies have shown the importance of performing a thorough and standardized handoff. ${ }^{13}$ It is unclear who provides safer care to patients: a well-rested resident who has to rely upon a handover, or a fatigued resident but with first-hand patient knowledge.

\section{Conclusion}

Residency training can be associated with reduced quality-oflife, increased burnout, and mental stress. However, reduction in resident duty hours has not been demonstrated to lead to harm or benefit for residents or for patients. In fact, reduced resident duty hours highlights new problems: work compression, increased handover, and decreased education.

Consecutive or total duty hours are not the only contributor to resident fatigue and burnout. Outside of clinical work, residents have research responsibilities, educational endeavours, and the opportunity to work extra shifts for additional income. Independently licensed physicians do not have restrictions on their work hours; as such, changes in duty hours in isolation do not reflect "real world" practice. Accordingly, residency training programs need to develop well-rounded fatigue management plans, not just duty-hour restriction.

Fatigue management includes ensuring a safe and supportive resident work environment, along with graded responsibility and adequate back-up. Time for sleep is important, as is attention to diet, exercise, support system, and mental health. These changes may require changes to how we configure our teams and deliver our education. This may include shifting towards focused exposure rather than sheer volume and a greater role for medical simulation. We must also recognize those at risk for burnout and in need of better coping strategies. There is clearly much work to be done. 


\section{References}

1. Nasca TJ, Day SH \& Amis ES. The new recommendations on duty hours from the ACGME Task Force. N Engl J Med 2010;363(2): e3.

2. Barger LK et al. Extended work shifts and the risk of motor vehicle crashes among interns. NEJM 2005; 352: 125-134.

3. Marcus C \& Loughlin G. Effect of sleep deprivation on driving safety in housestaff. Sleep 1996; 19: 763-766.

4. Arnedt JT et al. Neurobehavioural performance of residents after heavy night call vs after alcohol ingestion. JAMA 2005; 294: 1025-1033.

5. Lingenfelser TH et al. Young hospital doctors after night duty: their task-specific cognitive status and emotional condition. Med Educ. 1994; 28(6):566-572.

6. Fletcher KE et al. Systematic review: effects of resident work hours on patient safety. Ann Intern Med 2004; 141: 851-857.

7. Reed DA, Fletcher KE, Arora VM. Systematic review: association of shift length, protected sleep time, and night float with patient care, residents' health, and education. Ann Intern Med. 2010; 153(12): 829-842.

8. Ahmed $\mathrm{N}$ et al. A systematic review of the effects of resident duty hour restrictions in surgery: impact on resident wellness, training, and patient outcomes. Ann Surg. 2014; 259(6): 1041-1053.

9. Goitein L et al. The effects of work-hour limitations on resident well-being, patient care, and education in an internal medicine residency program. Arch Intern Med 2005; 165: 2601-2606.
10. Lefebvre DC. Resident physician wellness: a new hope. Acad Med 2012; 87: 598-602.

11. Auger KA et al. Better rested, but more stressed? Evidence of the effects of resident work hour restrictions. Acad Pediatr. 2012; 12(4): 335-343.

12. Sen $\mathrm{S}$ et al. Effects of the 2011 duty hour reforms on interns and their patients. JAMA Intern Med 2013; 173: 657-662.

13. Horwitz LI et al. Transfers of patient care between house staff on internal medicine wards: a national survey. Arch Intern Med. 2006; 166(11): $1173-1177$.

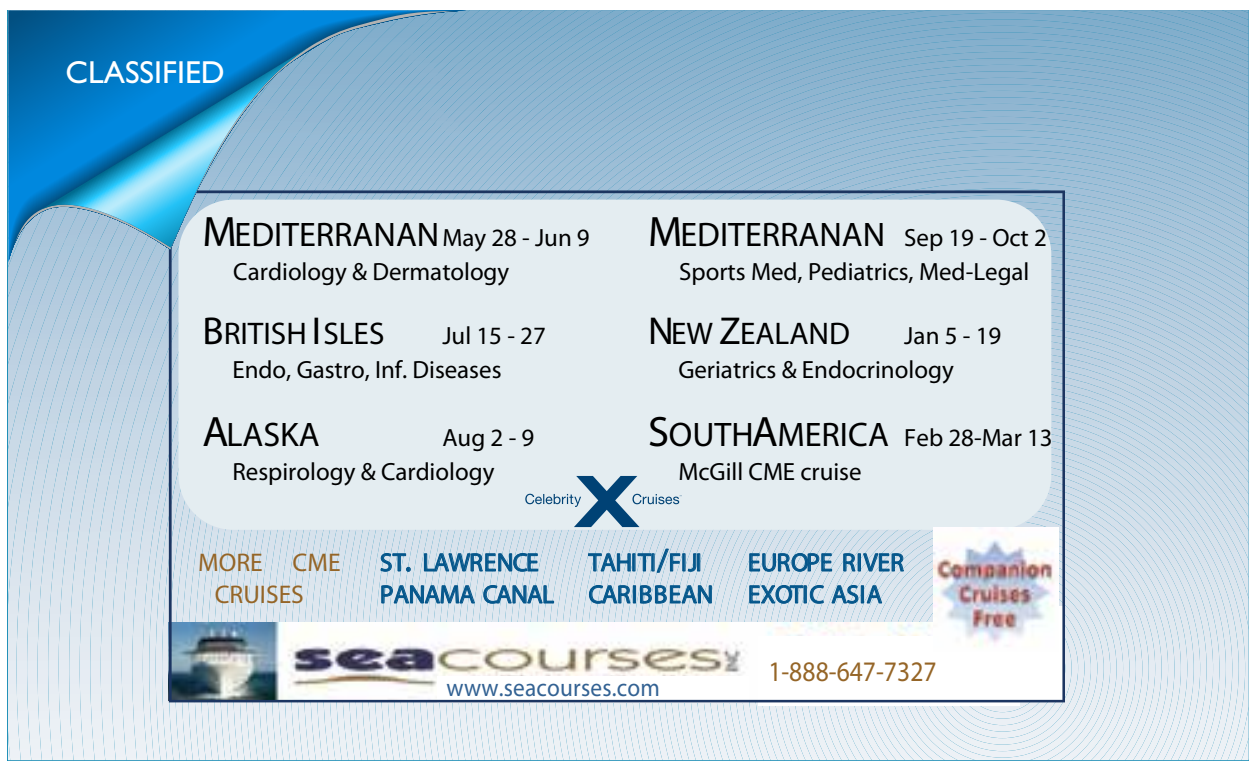

